\title{
Super sequential lateral growth of Nd:YAG laser crystallized hydrogenated amorphous silicon
}

\author{
Y. F. Tang a) and S. R. P. Silva \\ School of Electronic Engineering, Information Technology and Mathematics, University of Surrey, \\ Guildford GU2 7XH, United Kingdom \\ M. J. Rose \\ Department of Electronic Engineering and Physics, Dundee University, Carnegie Laboratory of Physics, \\ Dundee DD1 4HN, United Kingdom
}

(Received 3 July 2000; accepted for publication 6 November 2000)

\begin{abstract}
Lateral growth crystallization of hydrogenated amorphous silicon with single and multiple pulse excitation using a Nd:YAG laser at a wavelength of $532 \mathrm{~nm}$ and a $3 \mathrm{~ns}$ pulse width at a repetition of $10 \mathrm{~Hz}$ is shown. With single pulse crystallization, large grain sizes of the order of $1 \mu \mathrm{m}$ were obtained with an energy density $>400 \mathrm{~mJ} / \mathrm{cm}^{2}$, and these have been studied using transmission electron microscopy (TEM) and atomic force microscopy. We show that, by using extremely short (3 ns) multiple pulse excitation of significantly lower powers $\left(<150 \mathrm{~mJ} / \mathrm{cm}^{2}\right)$, than that used to crystallize amorphous silicon with single pulse excitation, a uniform growth of crystalline grains is observed. TEM gives evidence for lateral grain growth with multiple pulse crystallization at low energies. We suggest that a "super sequential lateral growth" mechanism is occurring. (C) 2001 American Institute of Physics. [DOI: 10.1063/1.1337627]
\end{abstract}

There is currently much interest in laser crystallization of amorphous silicon due to the electrically advantageous properties of nanocrystalline silicon for large area electronics. Much of the research has been carried out using excimer lasers at an ultraviolet (UV) wavelength. ${ }^{1,2}$ However, visible wavelength lasers could prove to be more advantageous for large area electronics due to a higher absorption length in amorphous silicon and because low cost glass is transparent in the visible wavelength but strongly absorbs at UV and visible UV wavelengths. ${ }^{3}$ Some research groups have demonstrated laser interference crystallization with the $532 \mathrm{~nm}$ laser line producing seed layers for seeded laser crystallization $^{3-6}$ using pulse widths down to $8 \mathrm{~ns}^{7}$

Much of the research to date has been performed using excimer laser crystallization of $a-\mathrm{Si}: \mathrm{H}$ to produce micro $(\mu c-\mathrm{Si}: \mathrm{H})$ or polycrystalline silicon (poly $\mathrm{Si})$. The high carrier concentrations and mobilities achieved in $\mu c-\mathrm{Si}: \mathrm{H} /$ poly $\mathrm{Si}$ means that the films are suitable for active devices such as solar cell or flat panel display driver electronics. In general, using single pulse excimer laser processing at a narrow energy window around $500 \mathrm{~mJ} / \mathrm{cm}^{2}$ gives super lateral growth (SLG) where an average grain size of a few $\mu \mathrm{m}$ is possible. In this case, an explosive crystallization front that nearly melts the silicon leaves behind fine grained poly $\mathrm{Si}$ when the temperature drops below the melting point. Since the grain size is limited due to the spontaneous nucleation of crystallites, Im and co-workers ${ }^{1}$ proposed sequential lateral solidification (SLS) to overcome this problem. In this case, successive laser pulses are used to melt the $a-\mathrm{Si}: \mathrm{H}$, which produces high quality poly $\mathrm{Si}$ due to the successive melting of the fine grain areas to form large crystalline sizes of several tens of microns.

In this letter, we discuss the differences observed be-

a)Electronic mail: yew.tang@eim.surrey.ac.uk tween single and multiple pulse crystallization of $a-\mathrm{Si}: \mathrm{H}$ using a Nd:YAG laser. We show that in our case a new mechanism, super sequential lateral growth (SSLG), produces good quality films at very low power densities where, even at 70 $\mathrm{mJ} / \mathrm{cm}^{2}$, it is possible to obtain single crystal grains of about $1 \mu \mathrm{m}$ if a large number of pulses are used.

The crystallization process was carried out using a Nd:YAG laser at $532 \mathrm{~nm}$ with a $3 \mathrm{~ns}$ pulse width at a repetition rate of $10 \mathrm{~Hz}$. The $a$-Si:H films used were $100 \mathrm{~nm}$ thick and prepared by the plasma enhanced chemical vapor deposition on Corning 7059 glass. Standard deposition parameters were used, with the gas flow of $40 \mathrm{sccm}$ of pure $\mathrm{SiH}_{4}$, at a chamber pressure of $150 \mathrm{mTorr}$ and at a temperature of $350{ }^{\circ} \mathrm{C}$ with a rf power of $6.5 \mathrm{~W}$. The $a-\mathrm{Si}: \mathrm{H}$ was crystallized in the near field $(<1 \mathrm{~m})$ with a relatively flat-top beam profile.

A series of samples was irradiated using a single pulse with varying energy density. Atomic force microscopy techniques were employed to investigate the surface morphology. The grain size and root-mean-square (rms) roughness dependence is shown in Fig. 1. Figure 1(b) shows the rms roughness of the surface, which maps the variation seen in the grain size closely as a function of laser energy density. The rms roughness remains at around $10 \%$ of the grain dimensions throughout the experimental range. Both of these parameters increase proportionately with energy density. In a similar study using a $532 \mathrm{~nm}$ laser line, Carius and co-worker ${ }^{4}$ did not observe any energy threshold. In our study, however, a clear step-like behavior at around 400 $\mathrm{mJ} / \mathrm{cm}^{2}$, is observed with a sudden increase in the grain size and rms roughness. Similar results have been observed by others using UV laser induced SLG of $a$-Si:H. ${ }^{8}$

TEM diffraction patterns taken of the irradiated area show single crystal diffraction with different orientations on the Nd:YAG single pulse crystallized films (Fig. 2). How- 


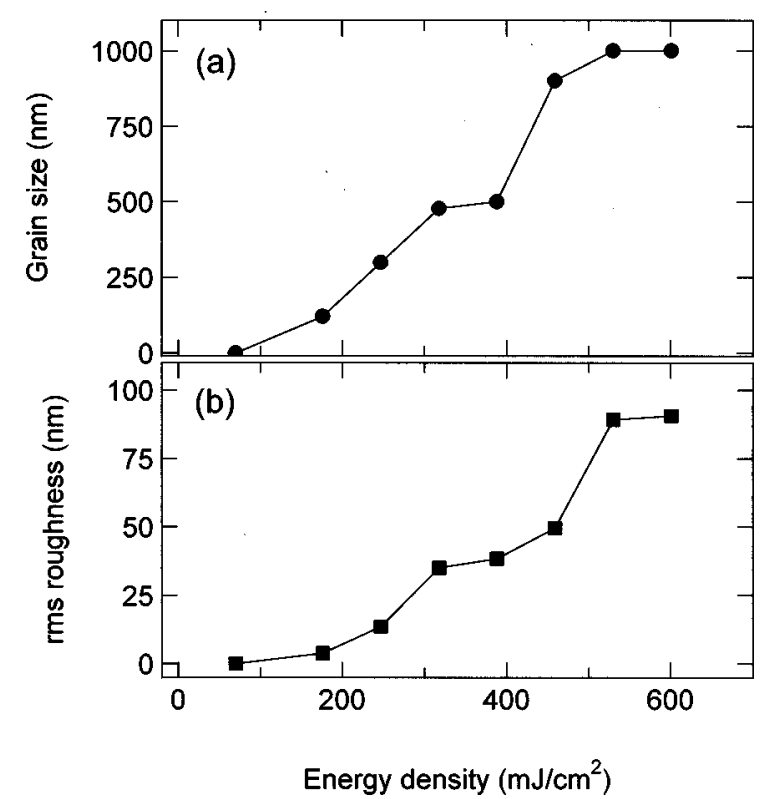

FIG. 1. Variation of (a) grain size (O) and (b) rms roughness ( $\mathbf{\square})$ vs energy density for single pulse laser crystallization at $532 \mathrm{~nm}$.

ever, it is noted that at the lower energy density (176 $\mathrm{mJ} / \mathrm{cm}^{2}$ ), an amorphous phase still remains as evidenced by the diffused rings in Fig. 2(a). The diffused amorphous rings are absent in Fig. 2(b) where the single pulse laser energy was increased to $530 \mathrm{~mJ} / \mathrm{cm}^{2}$. In this case, a good quality microcrystalline silicon film was created. No amorphous rings were present when a laser energy density in excess of $400 \mathrm{~mJ} / \mathrm{cm}^{2}$ was used. A micro-Raman scattering experiment performed on the laser crystallized region gives a well defined peak at $518 \mathrm{~cm}^{-1}$ with a full width at half maximum (FWHM) of $16 \mathrm{~cm}^{-1}$. The FWHM of a Raman signal from a commercial single crystal Si wafer $\left(14 \mathrm{~cm}^{-1}\right)$, when compared to the $16 \mathrm{~cm}^{-1}$ value, indicates a high quality material within the micro-Raman beam.

Multiple pulse crystallization was carried out with energy densities of 70,106 , and $123 \mathrm{~mJ} / \mathrm{cm}^{2}$ per pulse and with 10,1200 , and 3000 pulses per sample. A repetition rate of 10 $\mathrm{Hz}$ was used throughout the experiments and therefore it may be assumed that the $\mathrm{Si}$ molten front has reached an equilibrium before being exposed to successive laser pulses. The result of the grain size and rms roughness for these experiments is shown in Fig. 3. In multiple pulse crystallization, the roughness to grain size ratio is close to double $(20 \%)$ that observed with single pulses.

Both the rms roughness and the grain size increase as a function of laser power density as well as the number of pulses. However, in the three cases considered, the grain size reaches an optimum value in excess of $1 \mu \mathrm{m}$. A similar result is also observed for the rms roughness, which appears to indicate that, in the case of sequential laser crystallization, the lower the power a greater number of pulses is required to achieve large crystallite dimensions. This is a very interesting result as it indicates that it is possible to fully crystallize thin layers of the $a-\mathrm{Si}: \mathrm{H}$ in a controlled manner by using small packets of energy. It also alludes to the fact that the threshold observed in the single pulse crystallization process does not exist in the multiple pulse process that allows better

control of grain size formation.
The diffraction pattern taken from multiple pulse crys-
Downloaded 13 Oct 2010 to 131.227 .178 .158 . Redistribution subject to AIP license or copyright; see http://apl.aip.org/about/rights_and_permissions (a)

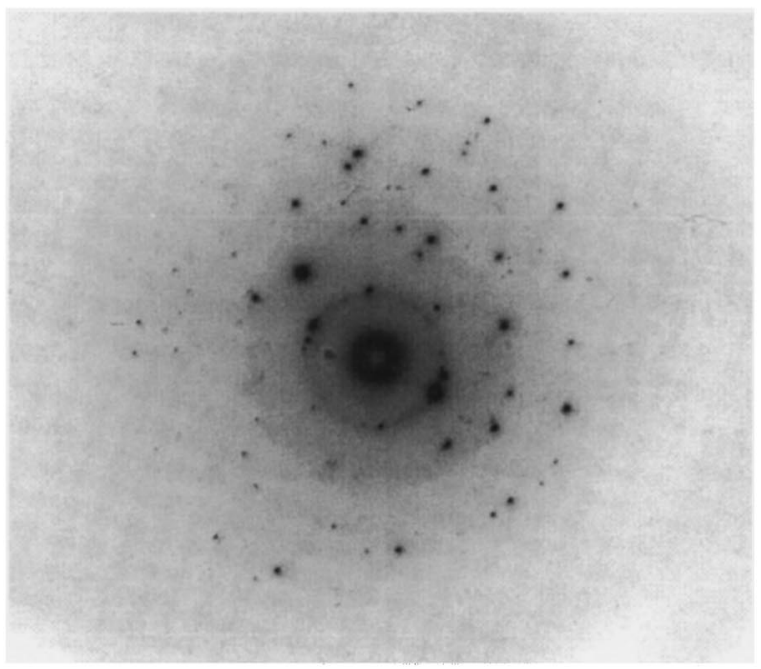

(b)

FIG. 2. TEM diffraction patterns for single pulse crystallization at $532 \mathrm{~nm}$ for energy densities of (a) $176 \mathrm{~mJ} / \mathrm{cm}^{2}$, and (b) $530 \mathrm{~mJ} / \mathrm{cm}^{2}$.

There is a significant and interesting variation in the grain size and rms roughness when the multiple pulse data is compared to single pulse crystallization. In the case of the single pulse data, a grain size of approximately $5 \mathrm{~nm}$ is observed when using an energy density close to $150 \mathrm{~mJ} / \mathrm{cm}^{2}$. In the multiple pulse crystallization process a steep increase in grain size is observed. For example, when an energy density of $123 \mathrm{~mJ} / \mathrm{cm}^{2}$ is used a grain size of $500 \mathrm{~nm}$ results after 10 pulses. This is significantly larger than if there was a linear relationship between the single pulse growth regime (where a crystal size of the order of $5 \mathrm{~nm} \times 10 \approx 50 \mathrm{~nm}$ would be expected) and the multiple pulse regime. The grain size increase is attributed to a SSLG regime. In this case, with each subsequent laser pulse a much faster growth process takes place, probably on the facets created during the previous pulse until a limiting value or plateau is reached, Fig. 3. Despite the rms roughness being significantly higher than in the single pulse case it appears that the large grain growth is uniform over the entire region rather than being faceted or oriented in one direction, i.e., polycrystalline film. The SSLG process is also observed for the other energies used in the multiple pulse excitation of $a-\mathrm{Si}: \mathrm{H}$.

The diffraction pattern taken from multiple pulse crys- 


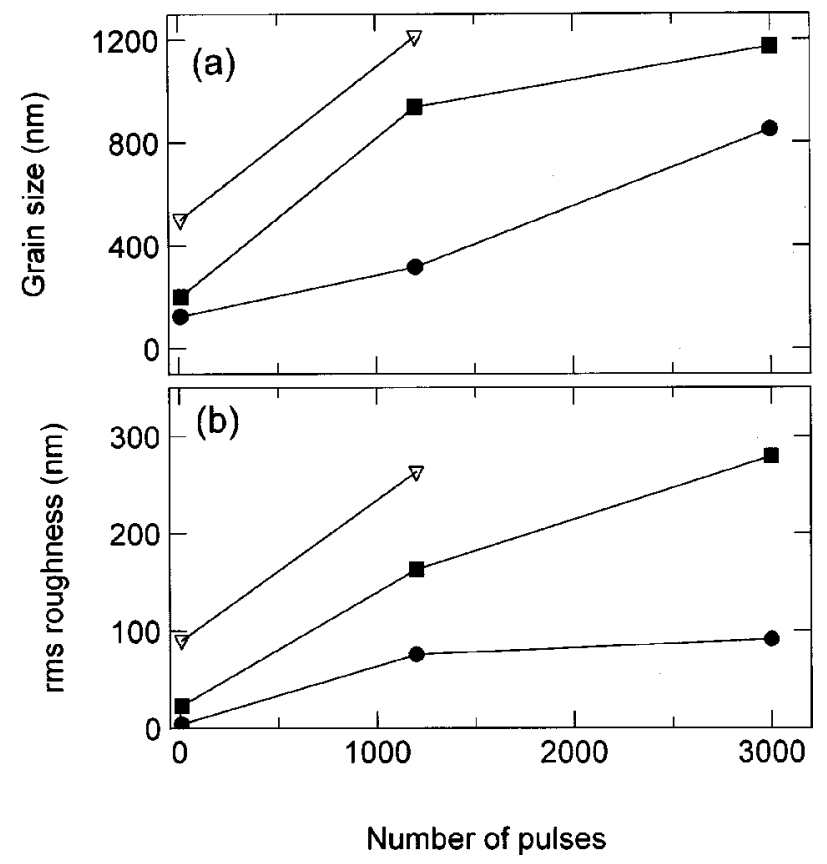

FIG. 3. Variation of the (a) grain size and (b) rms roughness vs number of pulses for multiple pulse laser crystallization using $532 \mathrm{~nm}$ with energy densities of $70(\bullet), 106(\mathbb{\square})$, and $123(\nabla) \mathrm{mJ} / \mathrm{cm}^{2}$.

tallized $a-\mathrm{Si}: \mathrm{H}$ is shown in Fig. 4. Figure 4, which is typical for the large grained multiple pulse irradiated films, shows sharp rings interspersed with well defined spots associated with Si that can be attributed to polycrystalline silicon. Raman analysis confirms the quality of the films. This gives us reason to believe that good quality crystalline films are in-

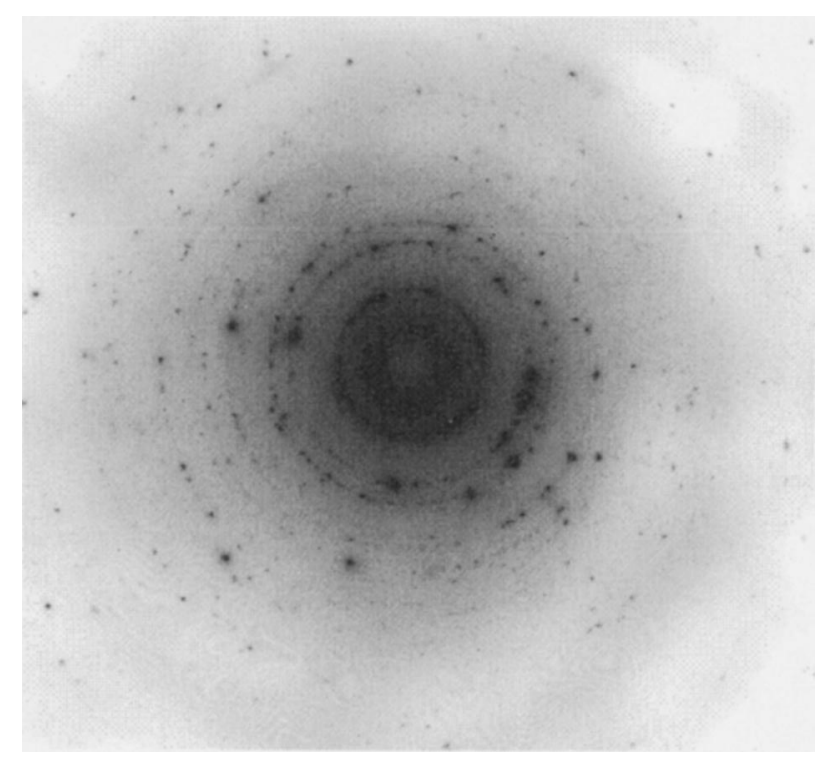

FIG. 4. TEM diffraction patterns showing a polycrystalline Si microstructure for a multiple pulse laser crystallization at $532 \mathrm{~nm}$ at an energy density of $106 \mathrm{~mJ} / \mathrm{cm}^{2}$ with 1200 pulses were used. deed obtained as a result of either SSLG or a hybrid SSLS and SLG process in the Nd:YAG irradiated films.

This result demonstrates that a new mechanism of crystallization is occurring in the $a-\mathrm{Si}: \mathrm{H}$ at a laser wavelength of $532 \mathrm{~nm}$. The interval between successive pulses is long compared to the quench time of laser crystallization of $a-\mathrm{Si}: \mathrm{H}$, therefore, the thin film experiences each laser pulse as an individual event, with temperature variations caused by each laser pulse being relatively similar. Mei and co-workers ${ }^{9}$ have postulated that an energy density slightly below the melting point of the $a-\mathrm{Si}: \mathrm{H}$ could help dehydrogenation and improve film surface morphology. A more recent study by Carius and co-workers ${ }^{4}$ has shown that changes in the thin film structure occur at $100 \mathrm{~mJ} / \mathrm{cm}^{2}$ with a $8 \mathrm{~ns}$ pulse width at $532 \mathrm{~nm}$. Their research provides evidence that lower energy densities can be used to reconstruct $a-\mathrm{Si}: \mathrm{H}$ thin films without phase changes. From our experiments, we show that the number of pulses required to crystallize amorphous silicon significantly affects the quality of film. We postulate that the crystallization model for the SSLG process begins on a thin localized layer on the surface of the $a-\mathrm{Si}: \mathrm{H}$ which subsequent pulses use as seed layers to crystallize laterally into a thin film. The absorption depth for $c$-Si $(500 \mathrm{~nm})$ is higher than that of $a-\mathrm{Si}: \mathrm{H}(100 \mathrm{~nm})$ for a wavelength of $532 \mathrm{~nm}^{10}$ and the crystallized Si should be able to withstand the laser pulse once it has reached its crystalline form. The short pulse width (3 ns) could create a stressed film and cause crystallization to occur at lower energy densities.

In conclusion, the multiple pulse crystallization technique has provided crystallization of $a-\mathrm{Si}: \mathrm{H}$ at a relatively low energy density. The surface roughness and grain size could be controlled within a large window. This has potential interest to technologists working on large area electronic devices such as solar cells and flat panel displays.

The authors acknowledge help received from EPSRC (GR/L77638) and Y.F.T. thanks CVCP for an ORS award.

${ }^{1}$ J. S. Im, M. A. Crowder, R. S. Sposili, J. P. Leonard, H. J. Kim, J. H. Yoon, V. V. Gupita, H. Jin Song, and H. S. Cho, Phys. Status Solidi A 166, 603 (1998)

${ }^{2}$ C. T. Angelis, C. A. Dimitriadis, M. Miyasaka, F. V. Farmakis, G. Kamarinos, J. Brini, and J. Stoemenos, J. Appl. Phys. 86, 4600 (1999).

${ }^{3}$ R. B. Bergmann, J. Kohler, R. Dassow, C. Zaczek, and J. H. Werner, Phys. Status Solidi A 166, 587 (1998).

${ }^{4}$ R. Carius, A. Wohillebe, L. Houben, and H. Wagner, Phys. Status Solidi A 166, 635 (1998).

${ }^{5}$ C. E. Nebel, S. Christiansen, H. P. Strunk, B. Dahlhelmer, U. Karrier, and M. Stutzmann, Phys. Status Solidi A 166, 667 (1998).

${ }^{6}$ G. Aichmayr, D. Toet, M. Mulato, P. V. Santos, A. Spangenberg, S. Christiansen, M. Albrecht, and H. P. Strunk, J. Appl. Phys. 85, 4010 (1999).

${ }^{7}$ W. Westlake and M. Heintze, J. Appl. Phys. 77, 879 (1995).

${ }^{8}$ J. S. Im, R. S. Sposili, and M. A. Crowder, Appl. Phys. Lett. 70, 3434 (1997)

${ }^{9}$ P. Mei, J. B. Boyce, M. Hack, R. Lujan, S. E. Ready, D. K. Fork, R. I. Johnson, and G. B. Anderson, J. Appl. Phys. 76, 3194 (1994).

${ }^{10}$ J. M. Potate and J. W. Mayer, Laser Annealing Semiconductor (Academic, New York, 1982). 